\title{
ON THE OPTIMAL CONTROL OF NATURAL RESOURCE \\ USE IN THE NEOCLASSICAL ECONOMIC FRAMEWORK*
}

\author{
A. Haurie and N. M. Hung \\ Ecole des Hautes Etudes Commerciales, Montróal.
}

Abstract:

One considers a class of neoclassical economic growth models where one commodity is a natural resource. Turnpike properties are proved for the finite horizon dynamic optimization problem and conditions are given for the existence of optimal programmes in the infinite horizon case. Some simple examples illustrate these findings.

Acknowledgements:

We would like to thank W.A. Brock who suggested the approach used for the existence problem. of course all remaining errors are ours.

\section{1.- Introduction.}

This paper is an attempt to deal rigorously with some fundamental mathematical problems arising from the consideration of natural resources in the neoclassical framework of optimal economic growth. The theory of optimal economic growth initiated by Ramsey 1928 and deveioped by many others has benefited considerably from an optimal control formulation, see for example She11 1967. Incidentally it posed two interesting problems which seem to be typical of these economic models.

The first one concerns the precise formulation of the Turnpike Property, see Cass 1966, the second one is the definition and the characterization of the optimality when the time interval considered is infinite and the formulation of existence conditions for such an optimal solution, see Koopmans 1965. It appeared that the two problems are linked together through the important role played in both problems by an optimal steady state suitably defined.

In the case of optimal exploitation of a natural resource, the Turnpike Property is well illustrated by the very simple model of optimal fish harvest studied by Cliff and Vincent 1973. Those authors showed that, for their particular model, any optimal trajectory on a sufficiently larce interval would contain a singular arc where the fish population is maintained at its level of maximal yield, this singular arc being independent of the initial and terminal conditions. When, in a given economy, more than one commodity, some of them being natural resources, enter into production, a similar property is expected to prevail. In section 2.2 it is shown that this is

* This research was supported by Canada Council (Grant 574-1122) and by the Ministère de I'Education du Québec DGES, FCAC. 
true: the Turnpike Property exhibited is expressed as a bound for the measure of the time spent away from the Von Neumann Set of the economy, a set which always contain the optimal steady state (s). When the cost of extraction does not depend on the importance of the stock of natural resource, an optimal steady state corresponds necessarily to the level of maximal yield for the natural resource.

When an exhaustible natural resource is essential in the production process, the concept of optimal steady states loses much of its significance since it corresponds to the degenerate situation with no production and no consumption. However another kind of property will be proved in section 2.3 which establishes a link between optimal programmes when a resource is exhaustible and the optimal steady state of a similar economy but where the natural resource is unexhaustible. Of course, that kind of Turnpike property is now dependent on the initial stock of the exhaustible resource.

In section 2.4 the existence of an optimal programme on the infinite horizon is proved. As noted by Solow 1975 one can have doubts that Time discount is defensible on the time scale appropriate for those models. The zero discount case is not a simple one since the use of the sufficient conditions of Arrow and Kurz 1970 is in general not possible and the sufficient conditions given by Rockafellar 1973 are not easily implemented. However, if there exists a unique optimal steady state the existence of an optimal programme is assured for the class of models under study. It is interesting to observe that the optimality concept introduced is stronger than those considered by Brock 1968 for a discrete time model and more recently by Halkin 1974 for an extension of the maximum principle.

Finally it should be noted that the method used to derive these results is quite general. Although the proofs make sometimes use of the peculiarities of the model defined in section 2.1, the same approach could be repeated for another model with more commodities. In fact these results could be obtained for a general class of convex control systems as shown by Rockafeliar 1973, Haurie 1976, Haurie and Brock 1976. 


\section{Turnpike and Existence Theorems for a Dynamic Economy with Natural Resource.}

In this section a model of an economy with a natural resource is introduced. We suppose that there exists a single consumption good which can reproduce itself in conjunction with the use of a commodity extracted from the environment: the natural resource. By not considering Time discount, this planning problem can be represented as being one of optimal control of an autonomous system. Our aim is to obtain a Turnpike property for optimal programmes and conditions for existence of an optimal programme when the planning horizon becomes infinite, under a minimal set of assumptions. The approach used is an adaptation to that particular model of the more abstract developments found in Haurie 1976 or Haurie and Brock 1976.

\subsection{The system under study.}

It is given by the following equations:

$$
\begin{aligned}
& \dot{W}=U(C) \\
& \dot{K}=I-\mu K, \quad H>0 \text { given } \\
& \dot{R}=G(R)-E \\
& I+C \leq F_{1}\left(K_{1}, E\right) \\
& \quad E \leq F_{2}\left(K_{2}, R\right) \\
& K_{1}+K_{2} \leq K \\
& 0 \leq R, 0 \leq C, 0 \leq I, 0 \leq E, 0 \leq K_{1}, 0 \leq K_{2}
\end{aligned}
$$

Where the state variables are: the accumulated utility for the system $W$, the stock of the reproducible composite commodity $K$ and the stock of the natural resource $R$. The control variables are: the consumption flow $C$, the investment $I$, the flow $E$ of natural resource into production, the part $K_{1}$ of the stock $k$ devoted to production of the composite commodity and the part $k_{2}$ devoted to the activity of extraction of the natural resource. $U($.$) is a concave functional defining the instantaneous$ utility of consumption, $F_{1}($.$) and F_{2}($.$) are concave and differentiable functionals$ describing respectively the production and extraction opportunities. $\mu$ is the depreciation rate and $G($.$) is a concave, differentiable functional describing the regene-$ ration process of the natural resource, as given for example by the Verhulst-Pear? êquation.

Various models recently proposed for the investigation of the optimal use of natural resources can be cast in the form of the system (2.1) - (2.7). This is particularly the case for those developed by Dasgupta and Heal 1975, Hung 1975, Plourde 1970.

The following terminology will be used: 
Definition 2.1:

a) A programme $\tilde{\pi}_{T}$ emanatina from $\left(K^{0}, R^{0}\right)$ is a map $\tilde{\pi}_{T}:[0, T] \rightarrow R^{8}\left([0, \infty) \rightarrow R^{8}\right.$ when $T=\infty$ ) such that:

(i) $\tilde{\pi}_{T}(t) \triangleq\left(\tilde{W}(t), \tilde{K}(t), \tilde{R}(t), \tilde{C}(t), \tilde{I}(t), \tilde{E}(t), \tilde{K}_{1}(t), \tilde{K}_{2}(t)\right)_{T}$

(ii) Almost everywhere on [0,T] Eqs. (2.1) - (2.3) are satisfied.

(iii) Everywhere on $[0, T)$ the constraints (2.4)-(2.7) are verified.

(iv) $\tilde{W}_{T}(0)=0, \tilde{K}_{T}(0)=K^{0}, \tilde{R}_{T}(0)=R^{0}$

b) To a given programme $\tilde{\pi}_{T}$ will be associated:

(i) The trajectory: $\tilde{y}_{T}:[0, T] \rightarrow R^{3}, \tilde{y}_{T}(t)=(\tilde{W}(t), \tilde{K}(t), \tilde{R}(t))_{T}$

(ii) The state path: $\tilde{x}_{T}:[0, T] \rightarrow R^{2}, \tilde{x}_{T}(t)=(\tilde{K}(t), \tilde{R}(t))_{T}$

c) A programme $\tilde{\pi}_{T}^{*}$ emanating from $\left(K^{0}, R^{0}\right)$ is optimal if for any other programme $\tilde{\pi}_{T}$ emanating from $\left(K^{0}, R^{0}\right)$ the following holds:

if $T<\infty, \tilde{W}_{T}^{*}(T) \geq \tilde{W}_{T}(T)$

if $T=\infty, \forall \varepsilon>0, \exists \tau, \forall t>\tau \quad \tilde{W}_{\infty}^{*}(t)>\tilde{W}_{\infty}(t)-\varepsilon$

This system satisfies the classical assumptions of optimal control theory as given by Lee and Markus 1967 and for $T<\infty$ fixed, the existence of an optimal programme is assured.

\subsection{Statement and Proof of the Turnpike Property.}

Consider the following optimization problem:

$$
\begin{aligned}
& \bar{U}=\operatorname{Max} U(C) \\
& \text { under the constraints }(2.4)-(2.7) \text { and } \\
& \dot{K}=I-\mu K \geq 0 \\
& \dot{R}=G(R)-E \geq 0
\end{aligned}
$$

This is a concave programming problem, thus if there exists a solution where none of the constraints (2.7) are active then there exists*a vector of Lagrange multipliers:

$$
\bar{\sigma} \triangleq(\bar{p}, \bar{q}, \bar{\lambda}, \bar{v}, \bar{\theta}) \geq 0
$$

such that for all vectors:

$$
Z \triangleq\left(K, R, C, I, E, K_{1}, K_{2}\right) \in R^{7}, Z \geq 0
$$

* See Mangasarian 1969. 
the following holas:

$$
\begin{aligned}
& \bar{U} \geq U(C)+\bar{p}(I-\mu K)+\bar{q}(G(R)-E)+\bar{\lambda}\left(F_{1}\left(K_{1}, E\right)-I-C\right) \\
& +\bar{v}\left(F_{2}\left(K_{2}, R\right)-E\right)+\bar{\theta}\left(K-K_{1}-K_{2}\right) \triangleq L(Z, \sigma) \quad(2.10)
\end{aligned}
$$

and for at least one $\bar{Z}$ satisfying the constraints:

$$
\bar{U}=U(\bar{C})
$$

Let $F$ denote the subset of a11 vectors $Z \geq 0$ for which the equality holds in (2.10), this is the Von Neumann Set of the economy. For a given $Z$ we are interested in the distance:

$$
d(Z, F) \triangleq \operatorname{Inf}\left\{\left\|Z-Z^{\prime}\right\|: Z^{\prime} \in F\right\} \text {. }
$$

Lemma 2.1:

Let $X$ be a given compact subset of $R^{7}$ then the following holds:

$$
\forall \varepsilon>0 \exists \delta>0 \text { s.t. } Z \in X \text { and } d(Z, F)>\varepsilon \Rightarrow \overline{0}-\delta>L(Z, \bar{\sigma})
$$

Proof: Similar to the one given by Atsumi 1965, McKenzie 1968 or Haurie 1976

Now the Turnpike Property can be established:

Theorem 2.1: Let us assume that:

a) There exists a compact subset $x \subset R^{7}$ such that, for any $T>0$, any programme $\tilde{\pi}_{T} \triangleq(\tilde{W}, \tilde{Z})_{T}$ is such that:

$$
\forall t \in[0, T] \quad \tilde{Z}_{T}(t) \in X
$$

b) There exists a programme $\tilde{\pi}_{\infty}^{+}$emanating from $\left(K^{0}, R^{0}\right)$ such that:

$$
\exists \bar{T}>0, \forall t \geq \bar{T} \quad U\left[\tilde{C}_{\infty}^{+}(t)\right]=\bar{U}
$$

Then for $T>\bar{T}$, any optimal programme will necessarily verify:

$$
m_{T}(\varepsilon)<\frac{1}{\delta}\left[\bar{U} \bar{T}+\bar{p}\left(\tilde{K}_{T}^{*}(T)-K^{0}\right)+\bar{q}\left(\tilde{R}_{T}^{*}(T)-R^{0}\right)\right]
$$

where $m_{T}(\varepsilon)$ is the Lebesgue measure of the subset of $[0, T]$ defined by:

$$
\mathbb{E}_{T}(\varepsilon) \triangleq\left\{t \in[0, T]: d\left(\tilde{z}_{T}^{*}(t), F\right)>\varepsilon\right\}
$$

Proof: Since $\tilde{\pi}_{T}^{*}$ is optimal, and since $U\left(\tilde{C}_{\infty}^{+}(t)\right)=\bar{U}$ for $t \geq \bar{T}$ one has:

$$
\tilde{W}_{T}^{*}(T) \geq \tilde{W}_{\infty}^{+}(T) \geq \bar{j}(T-\bar{T})
$$

Now, for an optimal programme the constraints (2.4)-(2.6) will always be active and thus, using $(2.10)-(2.12)$ and integrating between 0 and $T$ one obtains:

$$
\tilde{W}_{T}^{*}(T)<\bar{j} T-\bar{p}\left(K_{T}^{*}(T)-K^{0}\right)-\bar{q}\left(\tilde{R}_{T}^{*}(T)-R^{0}\right)-\delta m_{T}(\varepsilon)
$$

where $m_{T}(\varepsilon)$ has been defined as the measure of the set (2.15). Now (2.16) and (2.17) lead to: 


$$
m_{T}(\varepsilon)<\frac{1}{\delta}\left[\bar{U} \bar{T}+\bar{p}\left(\tilde{K}_{T}^{*}(T)-K^{0}\right)+\bar{q}\left(\tilde{R}_{T}^{*}(T)-R^{0}\right)\right]
$$

that is $(2.14)$.

\subsection{Interpretation of the Turnpike Property.}

2.3.1 The role of the compact set $X$ is essential in the proof of Theorem 2.1. Such a set will be naturally defined if there exists $K_{\max }>K^{0}$ and $R_{\max }>R^{0}$ such that under (2.2)-(2.7) one necessarily has the following implication

$$
K>K_{\text {max }} \text { and } R>R_{\text {max }} \Rightarrow \dot{K}<0 \text { and } \dot{R}<0
$$

2.3.2 The upper bound given in (2.15) means that, for large values of $T$, an optimal programme $\tilde{\pi}_{T}^{*}$ spends most of the period in the vicinity of the set $F$. When $F$ reduces to a single element $\bar{Z}$, the free disposal assumptions (2.4) and (2.5) imply that $\bar{Z}$ corresponds to the optimal steady state of the economy.

2.3.3 Let us write the Pontryagin necessary conditions* for an optimal trajectory $y_{T}^{*}$ of the system (2.1)-(2.7). Defining the Lagrangian $L(Z, \sigma)$ as in (2.10) the following holds when none of the constraints (2.7) are active:

$$
\begin{array}{ll}
\frac{\partial L}{\partial C}=U^{\prime}(C)-\lambda=0 & \frac{\partial L}{\partial I}=p-\lambda=0 \\
\frac{\partial L}{\partial E}=-q+\lambda \frac{\partial F_{1}}{\partial E}-\nu=0 & \frac{\partial L}{\partial K_{1}}=\lambda \frac{\partial F_{1}}{\partial K_{1}}-\theta=\frac{\partial L}{\partial K_{2}}=\nu \frac{\partial F_{2}}{\partial K_{2}}-\theta=0 \\
\frac{\partial L}{\partial K}=\theta-\mu p=-\dot{p} & \frac{\partial L}{\partial R}=q G^{\prime}(R)+\nu \frac{\partial F_{2}}{\partial R}=-\dot{q}
\end{array}
$$

while the constraints (2.2)-(2.6) are satisfied. Those conditions lend themselves to the standard interpretation in terms of marginal utility and marginal productivity. If there exists a unique solution to these equations when $\dot{p}=\dot{q}=\dot{K}=\dot{R}=0$, F reduces to a single element $\bar{Z}$ toward which the economy is driven.

2.3.4 The two limiting cases of an unexhaustible resource $\left(G(R) \equiv \infty, \partial F_{2} / \partial R \equiv 0\right)$ and of a non replenishable resource $(G(R) \equiv 0)$ deserve a particular attention.

In the first case the state equation (2.3) is no longer a constraint and (2.14) is replaced by:

$$
\mathrm{m}_{\mathrm{T}}(\varepsilon)<\frac{1}{\delta}\left[\overline{\mathrm{U}} \overline{\mathrm{T}}+\overline{\mathrm{p}}\left(\tilde{K}^{*}(T)-\mathrm{K}^{0}\right)\right]
$$

Now consider the second case. If the resource does not replenish itself, a steady state requires $E \equiv 0$ and it becomes clear that $F$ cannot reduce to a single element (any value for $R$ is compatible with $E=0$ ); furthermore the Turnpike property loses its significance since the optimal steady states correspond to a degenerate situation where the natural resource is not used in the economy.

for simplicity one will assume that $U($.$) is differentiable.$ 
However a different kind of "Turnpike" could be exhibited. First, we notice that the only difference between unexhaustible and non replenishable resources is that any positive rate of extraction can be maintained in the first case while no positive rate of extraction can be maintained in the second case. Nevertheless, if the initial stock is large enough, and if $\partial F_{2} / \partial R \equiv 0$, it may be possible to reach at time $\bar{T}$ the steady state $\bar{Z}$ corresponding to the unexhaustible case and then to maintain the extraction at level $\bar{E}$ until exhaustion of the resource at time $\hat{T}$. For all $T>\hat{T}$ an optimal programme $\tilde{\pi}_{T}^{*}$ will certainly verify.

$$
\tilde{W}^{\star}(T) \geq(\hat{T}-\bar{T}) \bar{U}
$$

Assuming that $\bar{Z}$ is unique and defining $m_{T}^{\prime}(\varepsilon)$ as the measure of the set:

$$
\Xi_{\top}^{\prime}(\varepsilon) \triangleq\left\{t \in[0, T]: d\left(\tilde{Z}_{T}^{*}(t), \bar{Z}\right)>\varepsilon\right\}
$$

a repetition of the arguments of Theorem 2.1 gives:

$$
m_{T}^{\prime}(\varepsilon)<\frac{1}{\delta}\left[\bar{U}(T-\hat{T}+\bar{T})+\bar{p}\left(\tilde{K}^{*}(T)-K^{0}\right)\right]
$$

For $T$ fixed, when $R^{0}$ increases $\hat{T}$ tends to be close to $T$ and thus (2.20) does not differ strongly from (2.18). The relation (2.19) says that when the initial stock of a non replenishable resource is large, an optimal programme will first drive the economy toward the steady state $\bar{Z}$ as if the resource was in infinite supply and only at the end of the period will the scarcity of the resource be taken seriousiy into account.

To illustrate that behaviour consider the following particular system:

$$
\begin{array}{lll}
\dot{W}=C & , \dot{K}=I-\mu K & , \dot{R}=-E \\
I+C \leq A K_{1}^{\alpha} E^{T-\alpha} & , A>0 \text { given }, \alpha \in[0,11 \text { given } \\
E \leq B \sqrt{K_{2}} & , B>0 \text { given }, \\
K_{1}+K_{2} \leq K & \\
0 \leq E, \quad 0 \leq K, \quad 0 \leq R, \quad 0 \leq I, \quad 0 \leq C, \quad 0 \leq K_{1}, \quad 0 \leq K_{2}
\end{array}
$$

The gradient of the Langrangian $L(Z, \sigma)$ is given by:

$$
\left.\begin{array}{lll}
\frac{\partial L}{\partial C}=1-\lambda & \frac{\partial L}{\partial I}=p-\lambda & \frac{\partial L}{\partial E}=-q-v+\lambda A(1-\alpha)\left(\frac{K_{1}}{E}\right)^{\alpha} \\
\frac{\partial L}{\partial K_{1}}=\lambda A \alpha\left(\frac{K_{1}}{E}\right)^{\alpha-1}-\theta & \frac{\partial L}{\partial K}=\frac{\nu B}{2 \sqrt{K_{2}}}-\theta \\
\frac{\partial L}{\partial K}=\theta-p \mu=-\beta & \frac{\partial L}{\partial R}=0=-\dot{q}
\end{array}\right\}
$$

If appears that an optimal trajectory will be composed of regular arcs where $C \equiv 0$ or $I \equiv 0$ and of a singular arc characterized by the condition: $p \equiv 1$.

Along such an arc the necessary conditions imply:

$$
\theta=u, \frac{K_{1}}{E}=\left(\frac{\alpha A}{\mu}\right)^{\frac{1}{1-\alpha}}, E=\frac{\nu B}{2 \mu}, q+v=A(1-\alpha)\left(\frac{K 1}{E}\right)^{\alpha}
$$


Since $q$ and $K_{7} / E$ are constants $v$ is also a constant and thus $E$ is a constant too. It appears that the singuiar arc is uniquely defined as a steady state and it would be easy to check that it corresponds to the optimal steady state when the resource is unexhaustible.

\subsection{Existence of an optimal programme on the infinite horizon.}

Theorem 2.2 : Under the assumptions of Theorem 2.1 and if $F$ reduces to the single element $\bar{z}$ then there exists an optimal programme $\tilde{\pi}_{\infty}^{*}$ emanating from $\left(K^{0}, R^{0}\right)$.

Proof: Define: $L^{\prime}(x, \bar{\sigma}) \triangleq \sup \left\{L(Z, \bar{\sigma}):\left(C, I, E, K_{7}, K_{2}\right) \geq 0\right.$, with $(2.4)-$ $(2.6)\}$.

$$
L_{0}(x) \triangleq L^{\prime}(x, \bar{\sigma})-\tilde{U} \leq 0
$$

$L_{0}(x)$ is non positive by $(2.10)$. Since there exists a programme $\tilde{\pi}_{\infty}^{+}$for which

$$
U\left[\tilde{C}_{\infty}^{+}(t)\right] \equiv \bar{U} \forall t \geq \tilde{T}
$$

One has certainly:

$$
\theta^{*}=\operatorname{Sup}\left\{\int_{0}^{\infty} L_{0}\left(\tilde{x}_{\infty}(t)\right) d t: \tilde{K}(0)=k^{0}, \tilde{R}(0)=R^{0}\right\}>-\infty
$$

where the sup is over all state paths $\tilde{x}_{\infty}$ with given initial values $K^{0}, R^{0}$. Now there exists a sequence $\left\{\tilde{x}_{\infty}^{n}\right\}_{n \in \mathbb{N}}$ of state paths such that:

$$
\lim _{n \rightarrow \infty} \int_{0}^{\infty} L_{0}\left(\tilde{x}_{\infty}^{n}(t)\right) d t=\theta^{*}
$$

By (2.27) and using the dominated convergence theorem one has also:

$$
\int_{0}^{\infty} \lim _{n \rightarrow \infty} L_{0}\left(\tilde{x}_{\infty}^{n}(t)\right) d t=\theta^{*}
$$

Varaiya 1967 has shown that for a system having the compactness and convexity properties assumed here the set of state paths $\tilde{x}_{\infty}$ emanating from $\left(K^{0}, R^{0}\right)$ is compact in the topology of uniform convergence on finite intervals. Thus the sequence $\left\{\tilde{x}_{\infty}^{n}\right\}_{n \in \mathbb{N}}$ has at least one cluster point $\tilde{x}_{\infty}^{*}$ for which, by $(2.23)$ and by the continuity of $L^{\prime}(., \bar{\sigma})$, one has:

$$
\theta^{*}=\int_{0}^{\infty} L_{0}\left(\tilde{x}_{\infty}^{*}(t)\right) d t
$$

To the state path $\tilde{x}_{\infty}^{*}$ corresponds a programme $\tilde{\pi}_{\infty}^{*}$. We have to verify that $\tilde{\pi}_{\infty}^{*}$ is optimal. Consider any other programme and form:

$$
E(T) \triangleq \int_{0}^{T}\left[U\left(\tilde{C}_{\infty}^{*}(t)\right)-U\left(\tilde{C}_{\infty}(t)\right] d t\right.
$$

By (2.10) and (2.22) the following holds:

$$
\begin{aligned}
E(T) \geq & \int_{0}^{T}\left[L_{0}\left(\tilde{x}_{\infty}^{*}(t)\right)-L_{0}\left(\tilde{x}_{\infty}(t)\right)\right] d t \\
& -\bar{p}\left[\tilde{K}_{\infty}^{*}(T)-\tilde{K}_{\infty}(T)\right] \\
& -\bar{q}\left[\tilde{R}_{\infty}^{*}(T)-\tilde{R}_{\infty}(T)\right]
\end{aligned}
$$


Assume that one does not have:

$$
\lim _{t \rightarrow \infty} \tilde{K}(t)=\bar{K} \quad \text { and } \quad \lim _{t \rightarrow \infty} \tilde{R}(T)=\bar{R}
$$

Thus one necessarily has $m_{\infty}(\varepsilon)=\infty$ and from (2.12):

$$
\int_{0}^{\infty} L_{0}\left(\tilde{x}_{\infty}(t)\right) d t=-\infty
$$

Now it is clear, by $(2.23)$ and $(2.27)$ that the following convergence property holds for the state path $\tilde{x}_{\infty}^{*}$

$$
\lim _{t \rightarrow \infty} \tilde{K}_{\infty}^{*}(t)=\bar{K} \quad \text { and } \quad \lim _{t \rightarrow \infty} \tilde{R}_{\infty}^{*}(T)=\bar{R}
$$

If $\tilde{x}_{\infty}$ converges toward $(\bar{K}, \bar{R})$, then for any $\varepsilon>0$, if $T$ is chosen large enough one has:

$$
E(T)>\int_{0}^{T}\left[L_{0}\left(\tilde{x}_{\infty}^{*}(t)\right)-L_{0}\left(\tilde{x}_{\infty}(t)\right)\right] d \tau-\varepsilon
$$

and therefore

$$
\int_{0}^{\infty}\left[U\left(\tilde{C}_{\infty}^{*}(t)\right)-U\left(\tilde{C}_{\infty}(t)\right)\right] d t \geq 0
$$

If $\tilde{x}_{\infty}$ is not converging toward $(\bar{K}, \bar{R})$, the terms $\vec{p} \tilde{K}_{\infty}(T)$ and $\bar{q} \tilde{R}_{\infty}(T)$ stay bounded while, by (2.27):

$$
\lim _{t \rightarrow \infty} \int_{0}^{T}\left[L_{0}\left(\tilde{x}_{\infty}(t)\right)-L_{0}\left(\tilde{x}_{\infty}(t)\right)\right] d t=\infty
$$

Therefore $(2.28)$ holds in this case too and $\tilde{\pi}_{\infty}^{*}$ is an optimal programme.

\subsection{Interpretation of the existence theorem.}

2.5.1 The unicity of the optimal steady state $\bar{z}$ is essential for the proof of Theorem 2.2. Thus the existence Theorem does not apply to the case of a non replenishable resource. Dasgupta and Heal 1975 us ing the approach suggested by Arrow and Kurz 1970 showed the existence of optimal programmes for a particular system with non replenishable resource and it does not seem to be possible to get more general results.

2.5.2 The method used to etablish the existence theorem is reminiscent of the approach of Pitchford 1974 to study optimal population growth. Mimicking his approach we would consider the problem:

$$
\operatorname{Max} \int_{0}^{T}[U(\tilde{C}(t))-\bar{U}] \text { dt with } \tilde{K}(T)=\bar{K}, \tilde{R}(T)=\bar{R}
$$

and under the constraints (2.2)-(2.7) with $T$ free. It is clear that a solution of this problem would define an optimal programme on the infinite horizon. From a theoritical point of view, the main weakness of this approach is that for most cases a solution does not exist with a finite value of $T$. Thus contrarily to what is claimed by Pitchford, the existence theorem of Lee and Markus does not hold in that case since it requires an upper bound for the admissible values for $T$.

2.5.3 As an illustration consider the following particular system:

$$
\dot{w}=C, \dot{K}=I-\mu k
$$




$$
\begin{aligned}
& \dot{R}=G R\left(R_{\max }-R\right)-E, G>0, R_{\max }>0 \text { given } \\
& I+C \leq A K_{1}^{\alpha} E^{1-\alpha}, A>0 \text { given }, \alpha \in[0,1] \text { given } \\
& E \leq B \sqrt{K_{2}}, B>0 \text { given, } K_{1}+K_{2} \leq K \\
& 0 \leq K, 0 \leq R, 0 \leq I, 0 \leq C, 0 \leq E, 0 \leq K_{1}, 0 \leq K_{2}
\end{aligned}
$$

The gradient of the Lagrangian as computed in (2.21) still holds excepted for the last component $\mathrm{aL} / \mathrm{aR}$ which becomes:

$$
\frac{\partial L}{\partial R}=G\left(R_{\max }-2 R\right) q=-\dot{q}
$$

A singular arc is still characterized by $p \equiv 1$ and thus one has:

$$
\begin{aligned}
& \theta=\mu, \frac{K_{1}}{E}=\left(\frac{\alpha A}{\mu}\right)^{\frac{1}{1-\alpha}}, E=\frac{\nu B}{2 \mu} \\
& q+\nu=A(1-\alpha)\left(\frac{K}{E}\right)^{\alpha}
\end{aligned}
$$

If $R=\frac{1}{2} R_{\max }$ one has $\dot{q}=0$ and the singular arc corresponds to the optimal steady state.

If $R \neq \frac{1}{2} R_{\max }$ then $\frac{K_{1}}{E}$ remains a constant and:

$$
\dot{E}=-\frac{B}{2 \mu} \dot{q}=\frac{G B}{2 \mu}\left(R_{\max }-2 R\right) q \quad\left\{\begin{array}{l}
>0 \text { if } R<\frac{R \max }{2} \\
=0 \text { if } R=\frac{R}{2} \\
<0 \text { if } R>\frac{R \max }{2}
\end{array}\right.
$$

It appears that the singular arc leads asymptotically to the optimal steady state.

\section{3.- Conclusion.}

The results of section 2 are obtained under the general assumptions of optimal control theory and concave programming. Therefore they hold even when the functions $U($.$) and F_{T}$ (.) are not differentiable. Moreover no assumption of homogeneity is required for the production function and even the Inada conditions are replaced by the weaker assumption of the existence of the compact set $x$.

From the economic viewpoint, the Turnpike Property is of great help in guiding the planner for the set-up of optimal economic policies. The planner's task is simplified once he has characterized the optimal steady state in the neighbourhood of which an optimal programme should stay for the most part of the planning period. Plourde 1970 obtained a qualitative result of the same kind for a one commodity mode1, the commodity being a replenishable resource.

When capital is introduced into the model, the characterization of optimal 
policies is possible by using the policy switching technique of Pitchford 1972 .

Such an analysis is done by Hung 1975. However the existence of optimal programmes on an infinite planning horizon was not correctly asserted in these earlier works. In this paper this gap is hopefully filled.

\section{References}

ARROW, K.J. and KURZ, M. 1970, Public Investment, The Rate of Return and Optimal Fiscal Policy, J. Hopkins Press.

ATSUMI, H. 1965, NeocTassical Growth and the Efficient Programm of Capital Accumulation, Review of Economic Studies 32: 127 - 136.

BROCK, W.A. 1970, On Existence of Weakly Maximal Programmes in a Multisector Economy, Review of Economic Studies 37: 275 - 280.

CASS, D. 1966, Optimum Growth in an aggregative model of capital accumulation: A Turnpike Theorem. Econometrica 34: 833 - 850.

CLIFF, E.H. and VINCENT T.L. 1973, An optimal Policy for a Fish Harvest, Journal of Optimization Theory and Applications 12: 485 - 496.

DASGUPTA, P. and HEAL G. 1975, The Optimal Depletion of Exhaustible Resources, Review of Economic Studies Symposium: 3 - 28.

HALKIN, H. 1974, Necessary Conditions for Optimal Control Probiems with Infinite Horizon, Econometrica 42: 267 - 273.

HAURIE, A. 1976, Optimal Control on an Infinite Time Horizon: The Turnpike Approach, Journal of Mathenatical Economics, to appear.

HAURIE, A. and BROCK, W.A. 1976, On Existence of OptimaT Trajectories over an Infinite Time Horizon, miméo, to appear.

HUNG, N.M. 1975, Essay on the Optimal Dynamic Exploitation of the Natural Resources and the Social Discount, Ph.D. Thesis Univ. of Toronto.

KOOPMANS, T.C. 1965, On the Concept of Optimal Economic Growth in The Economic Approach to Development Planning, North Holland.

LEE, E.B. and MARKUS, L. 1967, Foundations of Optimal Control Theory, J. Wiley.

MANGASARIAN, 0.1. 1969, Nonlinear Programming, McGraw-Hi11.

MC KENZIE, L.W. 1968, Accumulation Programmes of Maximum Uti1ity and the Von Neumman Facet, in J.N. Wolfe edit. Value Capital and Growth, Aldine.

PITCHFORD, J. 1972, Population and Optimal Growth, Econometrica, 40: $103-106$.

PLOURDE, C.G. 1970, A Simple Model of Replenishable Natural Resource Exploitation, American Economic Review, 60: 518 - 523.

RAMSEY, F.P. 1928, A Mathematical Theory of Saving, Economic Journal, 38: 543 - 559.

ROCKAFELLAR, T. 1973, Saddle Points of Hamiltonian Systems in Convex Problems of Lagrange, Journal of Optimization Theory and Applications, 12: $367-390$.

SHELL, K. edit. 1967, Essays in the Theory of Optimal Economic Growth, M.I.T. Press.

SOLOW, R.M. 1975, Intergenerational Equity and Exhaustible Resources, Review of Economic Studies, Symposium: $29-46$.

VARAIYA, P. 1967, On the Trajectories of a Differential System, in Mathematical Theory of Control, Balakrishnan, Newstadt edit, Academic Press. 